\title{
Ultrafiltration-thermisation du lait à la production : aspects bactériologiques
}

par

\author{
S. BENARD*, J. L. MAUBOIS* et A. TARECK** \\ (avec la collaboration de J. C. VACHOT et de J. SAMSON)
}

\section{INTRODUCTION}

L'équipement de traite constitue la source principale de contamination du lait à la production [16]. De par leur conception, les machines à traire, riches en recoins, coudes et discontinuités de surfaces (caoutchouc - verre - acier inoxydable), constituent des lieux particulièrement propices au développement bactérien. Leur généralisation chez les producteurs de lait s'est accompagnée d'une dégradation de la qualité bactériologique du lait cru [18].

Mais, il est également bien démontré qu'un nettoyage efficace et une stérilisation dans les règles de cet équipement peut abaisser le niveau de contamination à une valeur comprise entre 1000 et 10000 germes/ml [16].

La nature des germes apportés par l'installation de traite varie avec le type de détergent utilisé, la séquence de nettoyage suivie (température et durée) et l'état de surface des composants, notamment ceux en caoutchouc [13]. Le tank de stockage constitue quant à lui, une très faible source de contamination : moins d'une bactérie par ml selon Palmer [31]. Il en résulte donc que l'essentiel de la contamination du lait cru se situe en amont.

L'accroissement des distances moyennes entre producteurs et ateliers de traitement, consécutif au regroupement desdits ateliers, et le renchérissement du coût de l'énergie ont fortement incité à une réduction des fréquences de collecte. D'un ramassage quotidien voire bi-quotidien, on est passé à un ramassage tous les $2 \mathrm{j}$ ou même tous les $3 \mathrm{j}$ dans certaines zones. Cet allongement des temps de

\footnotetext{
* Laboratoire de Recherches de Technologie Laitière, I.N.R.A., 65, rue de Saint-Brieuc - 35042 Rennes cedex.

** Société Alfa-Laval, B.P. 55 - 78340 Les Clayes-sous-Bois.
} 
conservation du lait cru à la ferme trouvait sa justification dans la généralisation du refroidissement à température inférieure à $4^{\circ} \mathrm{C}$.

Or, dans le lait conservé à basse température, se développent les germes psychrotrophes appartenant pour la plupart au genre Pseudomonas [4].

Le temps de génération à $4^{\circ} \mathrm{C}$ de ces micro-organismes est de 6 à $8 \mathrm{~h}$, c'est-à-dire que leur population est multipliée par 10 en $24 \mathrm{~h}$ et par 4000 en $72 \mathrm{~h}$. En raison de ce développement spécifique, un changement de flore microbienne s'établit dans le lait cru réfrigéré. Les germes psychrotrophes qui ne représentent, en général, que 10 à $20 \mathrm{p} .100$ de la population microbienne du lait quittant la machine à traire [30] deviennent la population dominante du lait réfrigéré en des intervalles de temps variant de 1 à $6 \mathrm{j}$ selon la température de stockage $\left(6^{\circ} \mathrm{C}-4^{\circ} \mathrm{C}\right.$ ou $\left.0^{\circ} \mathrm{C}\right)[7,29,4]$. De plus, en se multipliant, ces micro-organismes synthétisent et libèrent des enzymes protéolytiques et lipolytiques très actives et fortement thermo-résistantes, bien que les cellules microbiennes qui les produisent soient, elles, détruites par un traitement thermique modéré, inférieur à la pasteurisation H.T.S.T. Par exemple, la valeur D (temps de traitement thermique nécessaire à une réduction de 90 p. 100 de l'activité enzymatique) d'une protéase extraite de Pseudomonas fluorescens est de $30 \mathrm{~s}$ à $150^{\circ} \mathrm{C}$ [2].

Une conservation prolongée à basse température du lait cru conduit donc, même pour des niveaux de contamination initiaux relativement faibles, à un développement important de micro-organismes psychrotrophes, et à une production significative d'enzymes. A cause de leur thermorésistance, ces lipases et protéases se retrouvent au niveau de toutes les chaînes de transformation laitière et sont reconnues comme responsables de nombreux défauts de fabrication : défaut de goût du lait pasteurisé [40], gélification du lait U.H.T. en cours de conservation [1], perte de 2 à 3 p. 100 du rendement en fabrication fromagère [5], défaut de rancidité dans les fromages et le beurre [21], lorsque la population en germes producteurs d'enzymes a dépassé la valeur seuil de $10^{6} / \mathrm{ml}$ [4].

La thermisation du lait sur le lieu de production, en sortie de machine à traire, a été proposée et étudiée comme palliatif à cette dégradation de la qualité bactériologique de la matière première lait, découlant du développement des germes psychrotrophes dans le lait cru conservé à basse température [40]. La qualité biochimique, bactériologique et organoleptique du lait traité à $74^{\circ} \mathrm{C} \pm 1^{\circ} \mathrm{C}$ et conservé $7 \mathrm{j}$ à $3^{\circ} \mathrm{C} \pm 1^{\circ} \mathrm{C}$, était considérée comme tout à fait satisfaisante et comparable à celle d'un lait témoin ayant subi ce même traitement thermique, mais après $48 \mathrm{~h}$ de conservation à l'état cru à $+3^{\circ} \pm 1^{\circ} \mathrm{C}$. Le nombre de germes mésophiles revivifiables après $7 \mathrm{j}$ de conservation à $3^{\circ} \mathrm{C}$ était inférieur à 10000 germes $/ \mathrm{ml}$.

Sur un autre plan, Maubois et Mahaut [23] proposaient l'implantation de la technologie d'ultrafiltration au niveau du producteur de 
lait dans le but d'améliorer l'économie de l'utilisation et de la transformation de la matière première laitière. Une telle proposition trouve sa justification dans le fait que, dans la grande majorité des pays producteurs de lait, une part dominante de l'eau, du lactose et des sels minéraux produits par le troupeau laitier n'est pas utilisée en alimentation humaine. Par exemple, en France, la proportion du lactose collecté, non utilisé en nutrition humaine est estimée à 75 p. 100 [15]. La mise en œuvre de la séparation de ces constituants (peu ou mal utilisés, donc peu ou mal valorisés par les humains) au niveau de l'exploitation laitière ou du groupe d'exploitations laitières exigent que soient satisfaits les impératifs suivants :

\section{Faisabilité technologique}

Proposée à la fin des années 60 pour le traitement du lactosérum [14] et du lait [24], la technique d'ultrafiltration sur membrane est maintenant largement employée par l'industrie laitière mondiale. Les surfaces de membrane installées dépassent $24000 \mathrm{~m}^{2}$ et sont en croissance exponentielle [12]. Les progrès accomplis en matière d'équipements et de membranes, notamment en matière de résistance thermique et physico-chimique, permettent d'envisager favorablement l'implantation de cette technologie dans un milieu de plus faible technicité que l'usine laitière.

\section{Valorisation sur place du perméat d'ultrafiltration}

Les travaux de Schingoethe [35] et de Thivend [36] ont montré que l'appétabilité de l'ultrafiltrat de lait ou de lactosérum était excellente chez le ruminant adulte et que la consommation sous forme liquide jusqu'à 50-60 $1 / \mathrm{j}$ /animal n'avait aucune influence négative sur la production laitière tant en ce qui concerne la quantité produite, qu'en ce qui concerne le taux protéique ou le taux butyrique (au contraire, ce dernier serait augmenté significativement). Selon Thivend [36] le coefficient d'utilisation digestive (C.U.D.) de l'ultrafiltrat se situe à $90 \mathrm{p}$. 100 et sa valeur énergétique à 0,95 unité fourragère par $\mathrm{kg}$ de matière sèche soit 0,05 unité fourragère au litre de produit sortant de l'équipement d'ultrafiltration.

\section{Utilisation et transformation sans contrainte du rétentat produit}

Le facteur de concentration (rapport du volume du lait produit au volume de rétentat) proposé par Maubois et Mahaut [23] et Maubois [27], de l'ordre de 2, constituait un compromis entre le gain micro-économique maximal et les exigences technologiques existant tant au niveau de l'opération d'ultrafiltration qu'en aval. La recherche d'un facteur de concentration élevé tel que celui étudié : 4 par Veillet-Poncet et al. [37] conduit certes à une plus grande utili- 
sation sur place du perméat et à une diminution supérieure des coûts de refroidissement, de stockage et de transport, mais elle entraîne aussi en raison de la chute des performances de l'ultrafiltre (nombre de litres de lait traités par heure et par $\mathrm{m}^{2}$ de membrane), un nettoyage et une désinfection plus ardus, donc plus difficiles à mettre en ouvre au niveau de l'exploitation laitière, un coût supérieur d'investissement en matériel d'ultrafiltration, une modification très importante des caractéristiques et du comportement rhéologiques du produit : les viscosités cinématiques d'un lait, d'un rétentat 2 et d'un rétentat 4 à $2^{\circ} \mathrm{C}$ sont respectivement de 3,$3 ; 6,5$ et $17 \mathrm{oP}$; les comportements rhéologiques du lait et du rétentat 2 sont quasiment newtoniens alors que celui du rétentat 4 est nettement rhéofluidifiant $[11,34]$. Une telle modification des caractéristiques physiques rend pratiquement inutilisables bon nombre d'équipements de pompage, de traitement d'échange thermique et de transformation actuellement en place dans les usines laitières et oblige, en outre, à une révision radicale des technologies de fabrication.

Le traitement et la transformation d'un rétentat ayant au plus une concentration égale à 2 sont au contraire largement pratiqués dans l'industrie laitière avec des matériels de traitement traditionnels (industrie de transformation du lait de brebis : le lait de cette espèce $a$, à peu de choses près, la composition biochimique d'un rétentat 2 de lait de vache) et des technologies conventionnelles largement éprouvées et d'incidences connues sur les qualités et la composition des produits terminaux $[33,22,26,9,17]$.

Mais un traitement du lait limité à une opération d'ultrafiltration laissait entier le problème de la qualité bactériologique de la matière première laitière et de ce fait s'avérait à la fois techniquement et économiquement d'intérêt limité [38]. Aussi, Maubois [27] a-t-il proposé de combiner cette opération d'ultrafiltration jusqu'à un facteur de concentration au plus égal à 2,0 avec une opération de thermisation pouvant aller jusqu'à une pasteurisation H.T.S.T. sur les lieux mêmes de production du lait (ferme ou groupe d'exploitations). Le présent travail, qui constitue la première partie des nombreuses études à entreprendre avant d'envisager la généralisation d'une telle opération dans les fermes laitières, avait pour objet d'étudier les aspects bactériologiques de la combinaison de ces deux traitements. Une ferme disposant d'une salle de traite avec transfert du lait ayant été équipée d'un matériel prototype, le développement bactérien était observé, dans un premier temps, sur des échantillons prélevés au niveau de cet équipement mais conservés au laboratoire. Dans un second temps, l'évolution de la flore microbienne des différents produits (laits et rétentats crus, rétentats thermisés) était observée en simulant les conditions réelles de production ; mélange en tank de collecte classique d'une fraction aliquote puis de la totalité des traites ultrafiltrées et thermisées. L'ensemble des expérimentations portait sur une période de 15 mois. 


\section{MATERIEL ET METHODES}

\section{Traitement du lait}

Le troupeau se composait de 50 vaches de race FFPN $\times$ Holstein.

Le lait recueilli dans l'unité terminale (capacité 100 1) de la machine à traire était dirigé sur un bac tampon dont la capacité variait entre 120 et $600 \mathrm{l}$ selon les essais. Il était aussitôt ultrafiltré en recirculation sur ce bac tampon à l'aide d'un module U.F.P. 2 (Alfa Laval - Suède) équipé de une ou deux cartouches de membrane de type fibre creuse PM 50 (Romicon - U.S.A.) ayant une surface unitaire de $2,48 \mathrm{~m}^{2}$. La température du lait soumis à ultrafiltration variait selon les essais, en fonction de la température extérieure, entre 25 et $30^{\circ} \mathrm{C}$. La durée de l'opération d'ultrafiltration variait en fonction de la quantité de lait traité et du facteur de concentration souhaité mais elle était toujours inférieure à $90 \mathrm{~min}$. L'ultrafiltrat était recueilli en bidons de matière plastique et distribué aussitôt aux vaches laitières.

Le rétentat était ensuite thermisé en continu à l'aide d'un équipement prototype «Microtherm » construit par la société Alfa-Laval (Suède) composé d'un échangeur-récupérateur à plaques, d'un système de production d'eau chaude et de régulation de température. La capacité de cet équipement était de $480 \mathrm{l} / \mathrm{h}$. La température de thermisation était fixée pour toutes les expérimentations à $72^{\circ} \mathrm{C} \pm 1^{\circ} \mathrm{C}$. Le temps de chambrage était maintenu, pour toutes les expérimentations réalisées, à $15 \mathrm{~s}$. La température du rétentat sortant de l'équipement de thermisation était de $16^{\circ} \mathrm{C} \pm 1^{\circ} \mathrm{C}$. Pour certains essais, il était fractionné, avec prélèvement d'une fraction aliquote de 501 mise à refroidir dans un tank de garde commercial d'une capacité globale de 2001 . Pour d'autres, il était dirigé en totalité vers le tank de collecte principal (capacité 1200 l) de la ferme.

Le nettoyage de l'ensemble de l'installation, à l'exception du module d'ultrafiltration, c'est-à-dire machine à traire, circuits de transfert du lait, Microtherm se faisait en place à l'aide d'un programmateur (Alfa-Laval, France) utilisant un détergent alcalin (Henkel) immédiatement après chaque expérimentation. Une fois par semaine, l'ensemble de l'installation était désinfecté à l'aide d'hypochlorite de sodium (150 $\mathrm{ppm}$ de $\mathrm{Cl}_{2}$ actif). Le nettoyage et la désinfection du module d'ultrafiltration étaient réalisés en utilisant successivement $50 \mathrm{I}$ d'une solution à 0,5 p. 100 de produit « Asepto liquide » (Henkel UR 260420) et 501 d'une solution d'hypochlorite de sodium (200 ppm de $\mathrm{Cl}_{2}$ actif). Une fois par mois, après démontage de l'échangeur la propreté des plaques était vérifiée visuellement.

\section{Conservation du lait, du rétentat cru et du rétentat thermisé}

Dans une première série d'expérimentations ( 8 mois), des échantillons (volume $100 \mathrm{ml}$ ) représentatifs du lait de la traite, des 
rétentats obtenus avant et après thermisation à $72^{\circ} \mathrm{C} \pm 1^{\circ} \mathrm{C}$, étaient conservés au laboratoire au bain-marie à $2^{\circ} \mathrm{C}-4^{\circ} \mathrm{C}-6^{\circ} \mathrm{C} \pm 0,5^{\circ} \mathrm{C}$ pendant $7 \mathrm{j}$.

Dans une seconde série d'expériences (4 semaines), 501 de rétentat thermisé provenant de la traite du matin étaient refroidis et cumulés sur une période de $4 \mathrm{j}$ dans un tank de 2001 réglé à $4^{\circ} \mathrm{C} \pm 1^{\circ} \mathrm{C}$ puis conservés dans ce récipient pendant une période de $4 \mathrm{j}$ supplémentaires.

Enfin, dans une troisième série d'expérimentations (3 semaines), la totalité du rétentat recueilli après chaque traite (matin et soir) était refroidi et cumulé pendant $4 \mathrm{j}$ dans le tank de garde réglé à $4^{\circ} \mathrm{C} \pm 1^{\circ} \mathrm{C}$.

L'ensemble des expérimentations a porté sur une période de 15 mois.

\section{Analyses biochimiques}

La teneur en matière sèche des échantillons de lait, de rétentat cru et thermisé était déterminée par dessiccation à l'étuve à $102^{\circ} \mathrm{C}$ $105^{\circ} \mathrm{C}$ pendant $7 \mathrm{~h}$.

La teneur en azote total était déterminée par la méthode Kjeldahl en utilisant un auto-analyseur Technicon. La teneur en protéines était calculée en utilisant le coefficient 6,38.

La teneur en matière grasse était déterminée par la méthode acidobutyrométrique Gerber.

\section{Analyses bactériologiques}

Les échantillons de lait, de rétentat cru et thermisé destinés à l'analyse bactériologique étaient prélevés aseptiquement, amenés au laboratoire dans un bain-marie contenant de la glace fondante et analysés dans les $3 \mathrm{~h}$ suivant le prélèvement.

Le dénombrement des germes aérobies mésophiles revivifiables était effectué sur milieu gélosé (PCA Difco $n^{\circ}$ 0479) après incubation à $30^{\circ} \mathrm{C}$ pendant $3 \mathrm{j}$.

Le dénombrement des germes psychrotrophes revivifiables était effectué sur le même milieu gélosé mais après incubation à $7^{\circ} \mathrm{C}$ pendant $10 \mathrm{j}$.

Le dénombrement des germes coliformes revivifiables était effectué selon la méthode de Ray [32] avec une incubation à $30^{\circ} \mathrm{C}$ pendant $24 \mathrm{~h}$.

Cette méthode a été retenue en raison de sa plus grande sensibilité, que nous avons vérifiée, en la comparant à la méthode classique par ensemencement sur gélose au rouge violet bile (Difco $\mathrm{n}^{\circ}$ 0012-01). 


\section{RESULTATS}

\section{Composition des rétentats et ultrafiltrats}

Une composition biochimique caractéristique représentative des produits mis en œuvre et obtenus au cours de nos expérimentations est donnée dans le tableau 1.

\section{TABLEAU 1}

Composition biochimique des laits, rétentats et ultrafiltrats

\begin{tabular}{l|c|c|c}
\hline & $\begin{array}{c}\text { Extrait sec } \\
(\mathrm{g} / \mathrm{kg})\end{array}$ & $\begin{array}{c}\text { Matière azotée } \\
\text { totale } \\
(\mathrm{g} / \mathrm{kg})\end{array}$ & $\begin{array}{c}\text { Matière grasse } \\
(\mathrm{g} / \mathrm{kg})\end{array}$ \\
\cline { 2 - 3 } & 128,0 & 33,2 & 44,3 \\
Lait cru & 191,6 & 61,5 & 80,0 \\
Rétentat thermisé & 54,3 & 2,2 & - \\
Ultrafiltrat & & & \\
\hline
\end{tabular}

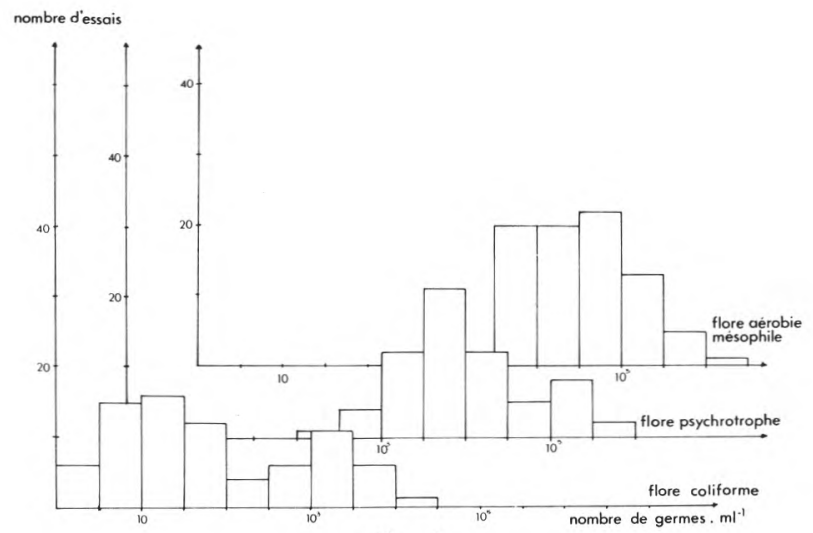

fig. 1

Répartition des flores aérobies mésophile, psychrotrophe et coliforme dans le lait cru suivant les essais.

\section{Bactériologie du lait avant traitement}

La qualité bactériologique du lait sortant de la machine à traire est caractérisée par les résultats rassemblés dans la figure 1. La 
variation observée de la population aérobie mésophile est assez élevée puisque les dénombrements effectués se situent entre 3000 et 1,2 million de germes $/ \mathrm{ml} .24$ p. 100 des traites analysées avaient une flore aérobie mésophile inférieure à 10000 germes $/ \mathrm{ml}$, et 55 p. 100 une flore comprise entre 10000 et 100000 germes. Dans un seul cas, une population supérieure à 1 million de germes était déterminée. La flore coliforme variait entre moins de 1 germe par ml et 20000 germes par $\mathrm{ml}$, mais pour plus de 50 p. 100 des laits analysés, représentait moins de 0,1 p. 100 de la flore aérobie mésophile. Les dénombrements de germes psychrotrophes des laits crus représentaient entre 0,1 et 125 p. 100 de la flore aérobie mésophile, ce qui est en accord avec les résultats obtenus par Bester [6].

\section{Développement bactérien au cours de l'ultrafiltration}

Les populations bactériennes des rétentats sortant de l'équipement d'ultrafiltration sont caractérisées par les résultats rassemblés dans la figure 2.

Le développement bactérien était apprécié comme proposé par Maubois et Mocquot [25], par la détermination du facteur F de multiplication microbienne avec

nombre de germes déterminé dans le rétentat

$\mathrm{F}=\overline{\text { nombre de germes dans le lait } \times \text { taux de concentration en volume. }}$

Pour l'ensemble de nos essais, les valeurs moyennes calculées étaient de :

$1,40 \pm 0,64$ pour la flore aérobie mésophile.

$1,46 \pm 0,92$ pour la flore psychrotrophe.

$2,22 \pm 1,55$ pour la flore coliforme.

Aucune relation n'a pu être mise en évidence entre la température d'ultrafiltration $\left(25^{\circ} \mathrm{C}\right.$ à $30^{\circ} \mathrm{C}$ ) et le développement des flores aérobies mésophile et psychrotrophe. Par contre, un coefficient de corrélation de 0,92 était calculé entre la température d'ultrafiltration et le développement de la flore coliforme.

\section{Effet du traitement thermique sur la flore}

Faute de moyens de prélèvements adéquats sur l'appareillage "Microtherm ", (notamment robinet de prélèvement d'échantillons en sortie de chambreur), l'effet du traitement thermique était apprécié de façon globale, c'est-à-dire après montée du rétentat de $25^{\circ} \mathrm{C}$. $30^{\circ} \mathrm{C}$ à $72^{\circ} \mathrm{C}$, maintien $15 \mathrm{~s}$ à cette température et refroidissement à $15^{\circ} \mathrm{C}-17^{\circ} \mathrm{C}$, donc en prenant en compte les recontaminations éventuelles liées à cette phase de refroidissement (eau de " pousse » et premiers litres de rétentat insuffisamment chauffés).

La destruction des germes par le traitement thermique retenu lors de la réalisation des expérimentations $\left(72^{\circ} \mathrm{C} \pm 1^{\circ} \mathrm{C}-15 \mathrm{~s}\right)$ est 


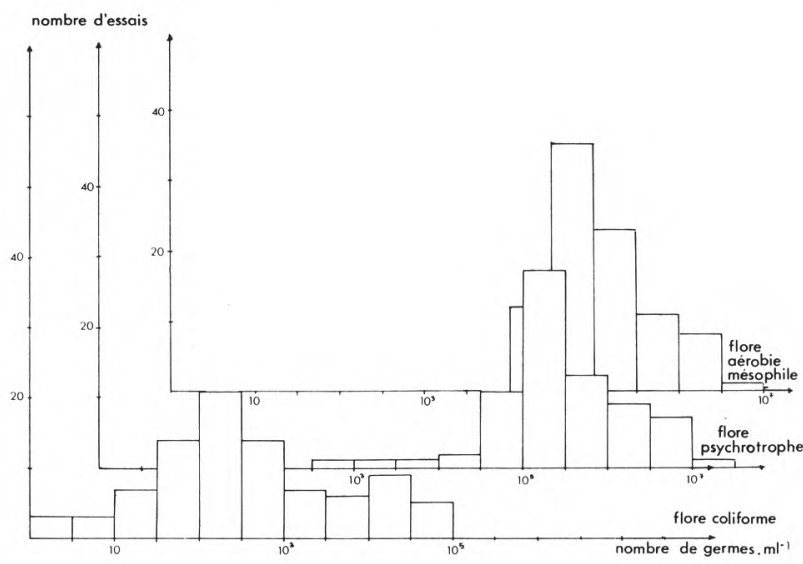

fig. 2

Répartition des flores aérobies mésophile, psychrotrophe et coliforme dans le rétentat cru 1,5-2,0, suivant les essais.

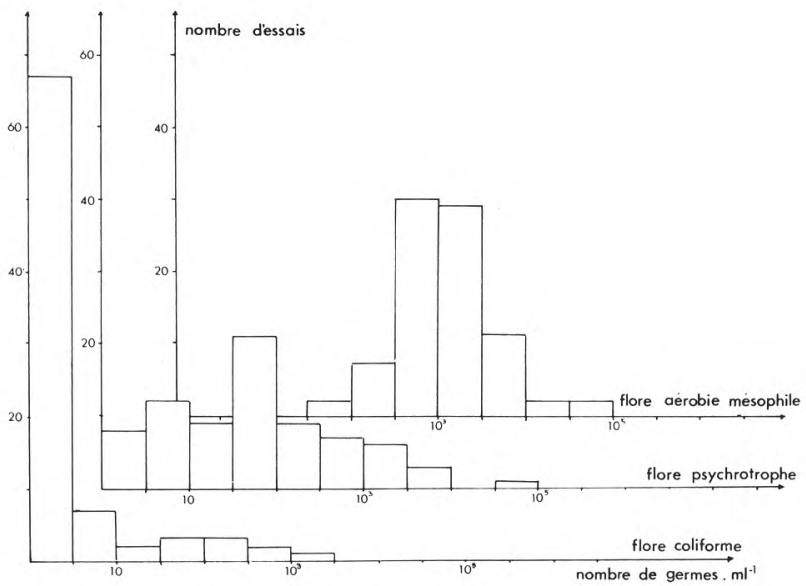

fig. 3

Répartition des flores aérobies mésophile, psychrotrophe et coliforme dans le rétentat $1,5-2,0$ thermisé $\left(72^{\circ} \mathrm{C} \pm 1^{\circ} \mathrm{C} / 15 \mathrm{~s}\right)$ suivant les essais.

illustrée par la comparaison des résultats rassemblés dans les figures 2 et 3 . La réduction décimale moyenne observée pour la flore mésophile était de $1,66 \pm 0,30$. 


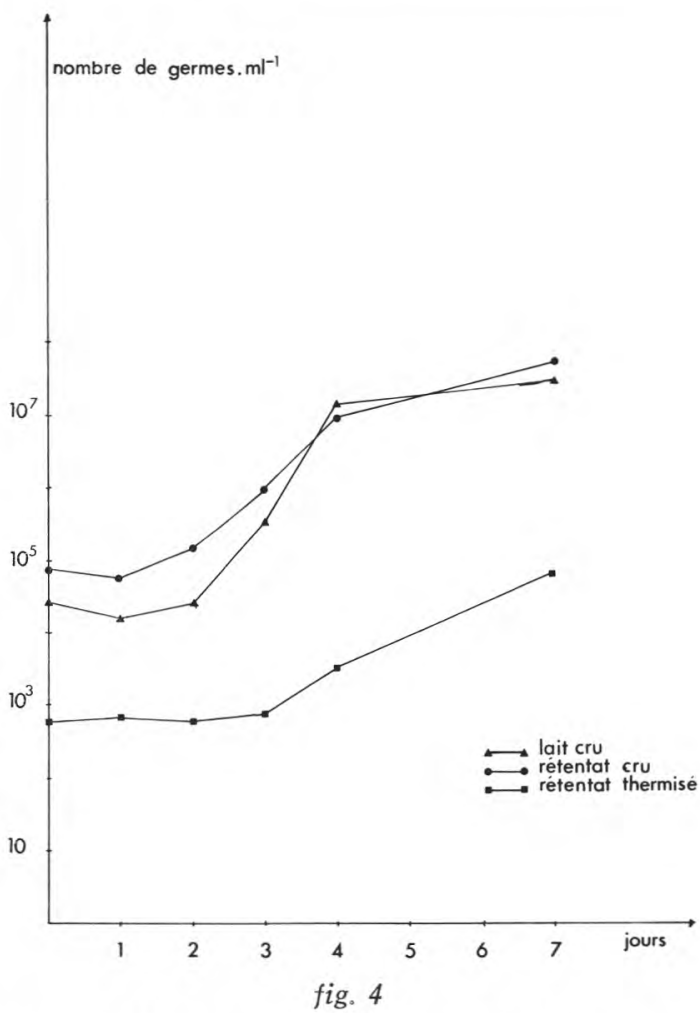

Evolution de la flore aérobie mésophile d'un lait et de rétentats $2 \mathrm{cru}$ et thermisé $\left(72^{\circ} \mathrm{C} \pm 1^{\circ} \mathrm{C} / 15 \mathrm{~s}\right)$ au cours de la conservation à $2^{\circ} \mathrm{C}$.

La flore coliforme après traitement thermique était inférieure à 15 germes/ml dans la quasi-totalité des dénombrements. Moins de 1 germe par ml était dénombré à la dilution 0 dans 83 p. 100 des expérimentations. Les deux seuls dénombrements significatifs d'une recontamination coliforme du rétentat dans la section de refroidissement du «Microtherm » étaient dus à une pollution accidentelle.

La flore psychrotrophe était détruite de façon très variable selon les expérimentations. La réduction décimale oscillait entre 2,5 et 3,5 en moyenne, mais avec des écarts parfois nettement plus accentués au niveau des déterminations individuelles. Par exemple, des rétentats ayant avant thermisation des populations psychrotrophes identiques: soit 11200 et 11300 ou bien 460000 et 470000 germes par ml présentaient respectivement après thermisation les populations suivantes : 1120 et moins de 10 germes par $\mathrm{ml} ; 400$ et absence de germes dans $2 \mathrm{ml}$. 


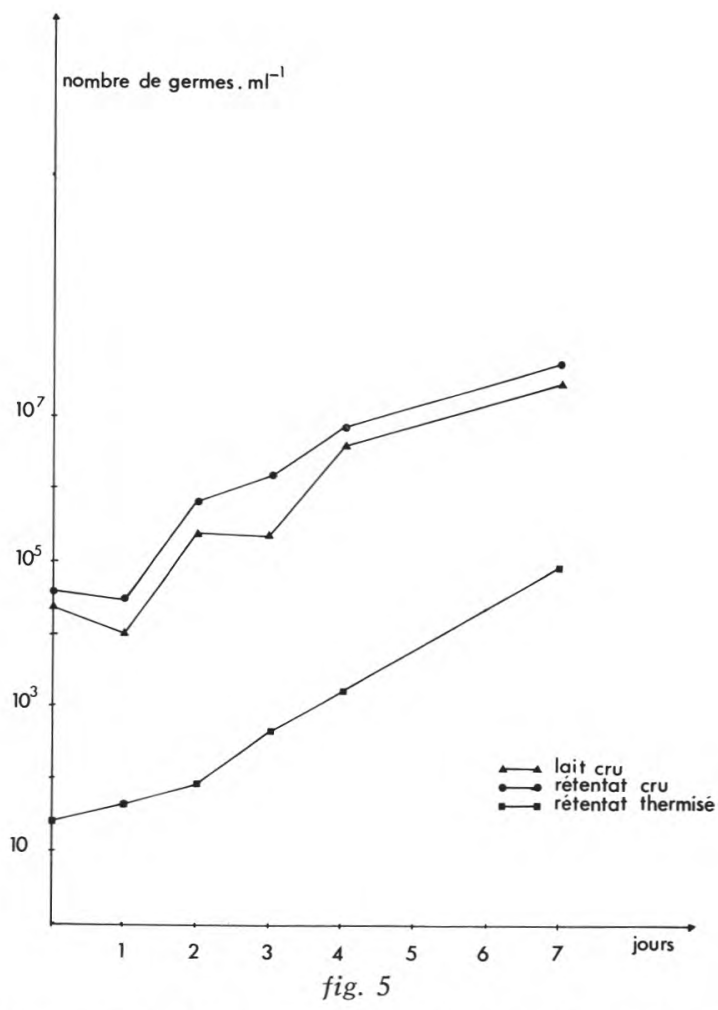

Evolution de la flore psychrotrophe d'un lait et de rétentats 2 cru et thermisé $\left(72^{\circ} \mathrm{C} \pm 1^{\circ} \mathrm{C} / 15 \mathrm{~s}\right)$ au cours de la conservation à $2^{\circ} \mathrm{C}$.

\section{Conservation du lait, des rétentats crus et thermisés au laboratoire}

Les figures 4, 6 et 8 illustrent l'évolution des populations aérobies mésophiles dans le lait $\mathrm{cru}$, le rétentat $\mathrm{cru}$ et le rétentat thermisé aux trois températures étudiées, soit respectivement $2^{\circ} \mathrm{C}-4^{\circ} \mathrm{C}$ et $6^{\circ} \mathrm{C}$.

Les figures 5, 7 et 9 sont représentatives de l'évolution de la flore psychrotrophe au sein des mêmes produits, conservés dans les mêmes conditions.

L'évolution des flores aérobies mésophiles et psychrotrophes était tout à fait similaire dans le lait cru et le rétentat cru, quelle que fût la température de conservation. Comme attendu, les croissances observées pour la flore aérobie mésophile étaient très dépendantes de ces températures de conservation, elles étaient voisines ou supérieures à $1 \log / \mathrm{j}$ à $6^{\circ} \mathrm{C}$, comprises entre 0,5 et $1 \log / \mathrm{j}$ à $4^{\circ} \mathrm{C}$, quasi- 


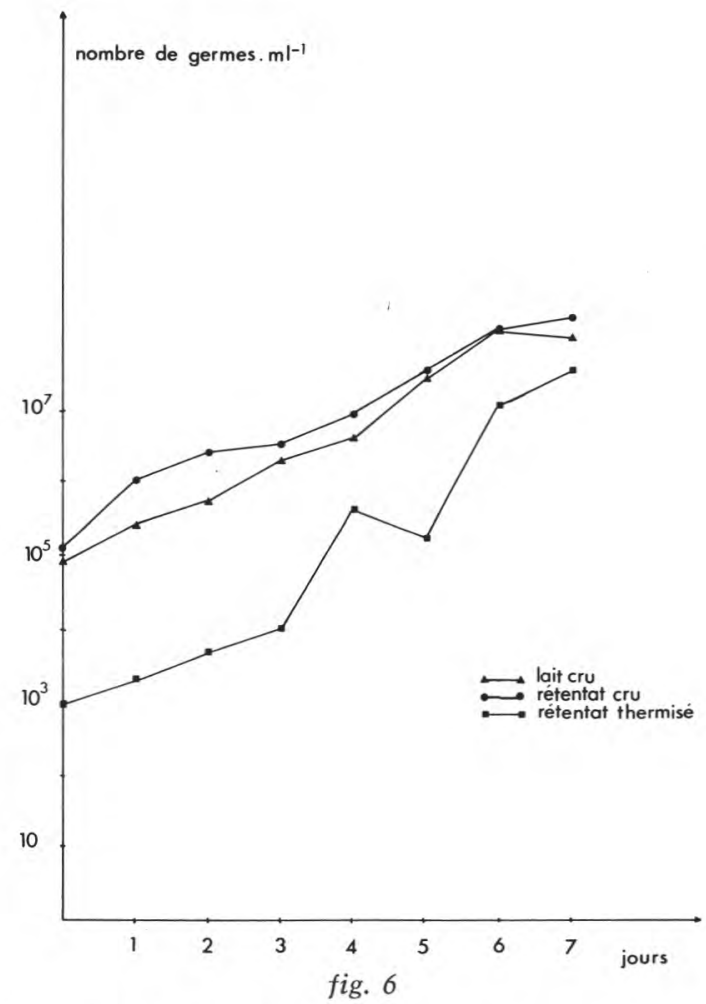

Evolution de la flore aérobie mésophile d'un lait et de rétentats $2 \mathrm{cru}$ et thermisé $\left(72^{\circ} \mathrm{C} \pm 1^{\circ} \mathrm{C} / 15 \mathrm{~s}\right)$ au cours de la conservation à $4^{\circ} \mathrm{C}$.

ment nulles pendant $48 \mathrm{~h}$ à $2^{\circ} \mathrm{C}$ pour atteindre ensuite $1 \mathrm{log} / \mathrm{j}$ au $3^{\text {e }}$ jour de conservation. Les croissances observées pour la flore psychrotrophe étaient très voisines de celles observées pour la flore aérobie mésophile à $6^{\circ} \mathrm{C}$ et $4^{\circ} \mathrm{C}$. Par contre, à $2^{\circ} \mathrm{C}$, une croissance voisine de $1 \mathrm{log} / \mathrm{j}$ était observée dès le $2^{\mathrm{e}}$ jour de conservation.

L'évolution des flores aérobies mésophiles et psychrotrophes dans le rétentat thermisé conservé à $6^{\circ} \mathrm{C}$ se caractérisait pour une lagphase de 24 h suivie d'une croissance rapide de 1,5 à $2 \mathrm{log} / \mathrm{j}$ jusqu'au $5^{\mathrm{e}}$ jour, durée de conservation après laquelle les populations dénombrées étaient identiques à celles dénombrées dans les produits crus. La conservation à $4^{\circ} \mathrm{C}$ réduisait nettement les croissances de flore aérobie mésophile $(0,3 \log / \mathrm{j}$ pendant $72 \mathrm{~h}$ puis 0,5 à $1,0 \mathrm{log} / \mathrm{j}$ pendant les $3 \mathrm{j}$ suivants $)$ et psychrotrophe $(0,3 \mathrm{log} / \mathrm{j}$ pendant $48 \mathrm{~h}$ puis $1,2 \mathrm{log} / \mathrm{j}$ pendant les $3 \mathrm{j}$ suivants). A $2^{\circ} \mathrm{C}$, la flore aérobie mésophile dénombrée restait stable pendant $72 \mathrm{~h}$, elle croissait ensuite de $0,4 \mathrm{log} / \mathrm{j}$. Même après $7 \mathrm{j}$ de conservation, la population dénombrée était infé- 


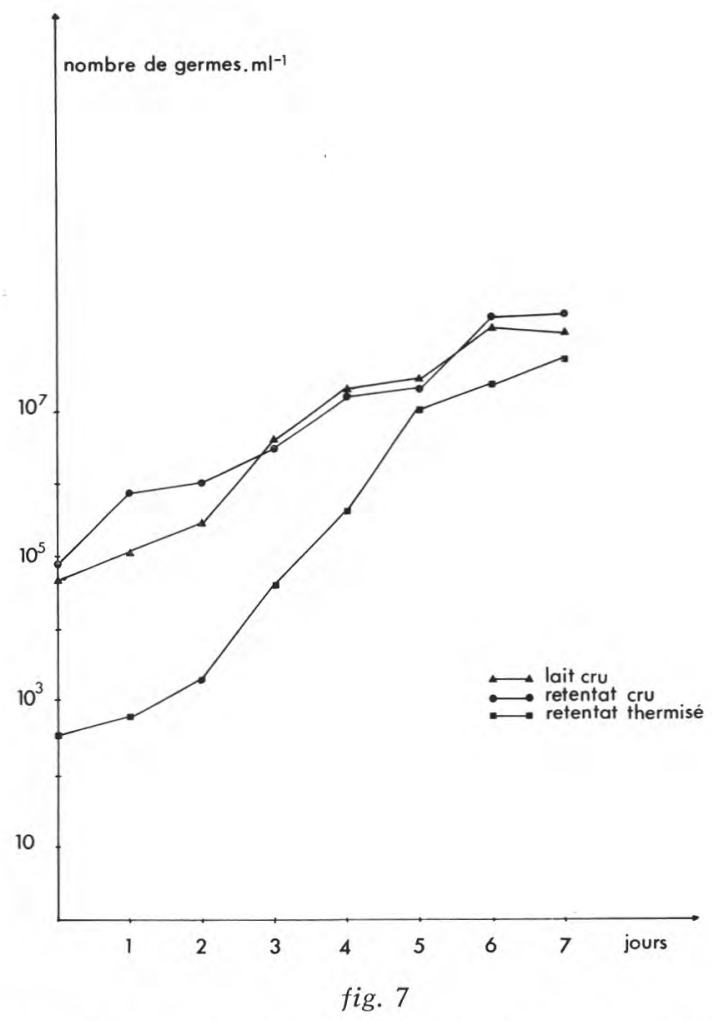

Evolution de la flore psychrotrophe d'un lait et de rétentats 2 cru et thermisé $\left(72^{\circ} \mathrm{C} \pm 1^{\circ} \mathrm{C} / 15 \mathrm{~s}\right)$ au cours de la conservation à $4^{\circ} \mathrm{C}$.

rieure à 100000 germes $/ \mathrm{ml}$. La croissance de la flore psychrotrophe variait de $0,25 \mathrm{log} / \mathrm{j}$ (premières $48 \mathrm{~h}$ de conservation) à $0,5 \mathrm{log} / \mathrm{j}$ (5 jours suivants).

L'évolution observée de la flore coliforme dépendait, comme cela était attendu, du niveau de population initiale et de la température de conservation des échantillons. A $6^{\circ} \mathrm{C}$, la croissance dans les produits crus et thermisés était égale ou supérieure à $1 \mathrm{log} / \mathrm{j}$. La population du rétentat thermisé rejoignait celles des produits crus vers le $5^{\mathrm{e}}$ jour de conservation. A $4^{\circ} \mathrm{C}$, la croissance était ralentie pour les trois types de produits (de l'ordre de 0,5 à $0,6 \log / \mathrm{j}$ ) mais la population du rétentat thermisé ne représentait, quelle que soit la durée de conservation, que 1 à 3 p. 100 des populations dénombrées dans les produits crus. A $2^{\circ} \mathrm{C}$, la croissance était très faible jusqu'au $6^{\mathrm{e}}$ jour dans les produits crus et thermisés. Ensuite, une croissance de l'ordre de $1 \mathrm{log} / \mathrm{j}$ était observée dans le lait et le rétentat crus. 


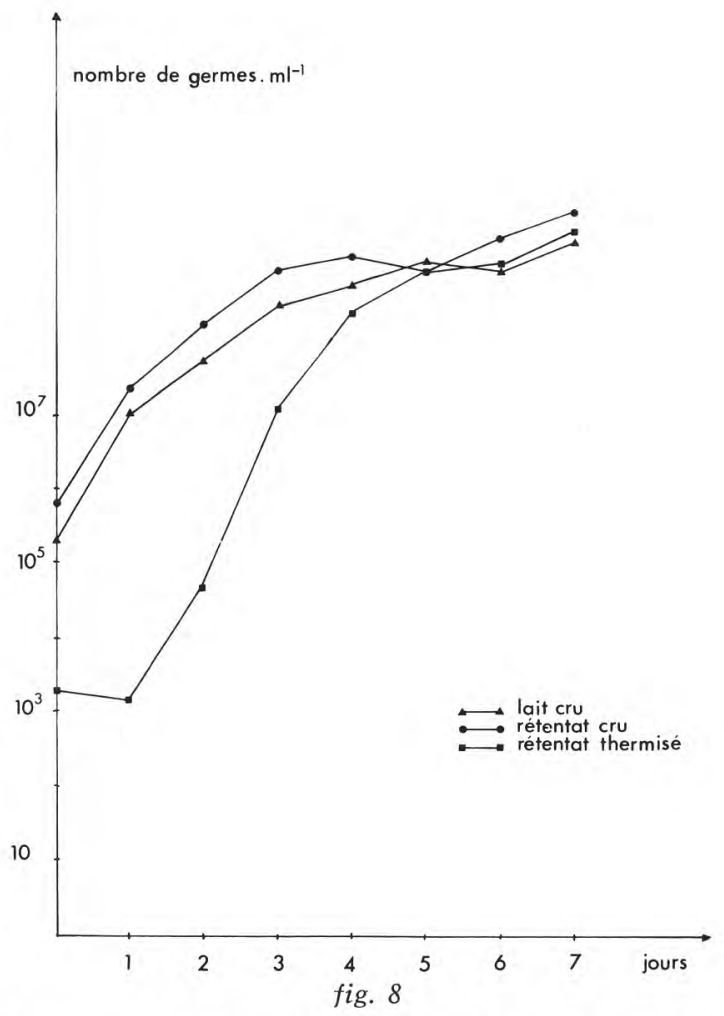

Evolution de la flore aérobie mésophile d'un lait et de rétentats $2 \mathrm{cru}$ et thermisé $\left(72^{\circ} \mathrm{C} \pm 1^{\circ} \mathrm{C} / 15 \mathrm{~s}\right)$ au cours de la conservation à $6^{\circ} \mathrm{C}$.

\section{Conservation du rétentat thermisé en tank de stockage à la ferme}

Les fig. 10 et 11 caractérisent l'évolution des flores aérobie mésophile et psychrotrophe dans le mélange de rétentats thermisés additionnés traite du matin après traite du matin (fig. 10) ou traite après traite (fig. 11) pendant $4 \mathrm{j}$.

La flore aérobie mésophile des laits crus était, en moyenne, dans les deux séries d'expérimentations de 25000 germes $/ \mathrm{ml}$. Elle était multipliée en moyenne par 2,8 (variation totale 1,6 à 4,0) par l'opération d'ultrafiltration en raison de la multiplication microbienne liée à la durée de cette opération (facteur 1,4) et de l'effet de concentration lié à l'opération elle-même (facteur 2). Le traitement thermique réalisé sur le rétentat cru réduisait cette flore par un facteur égal à environ deux unités logarithmiques, ce qui conduisait à une population moyenne voisine de 1000 germes $/ \mathrm{ml}$. La croissance observée 


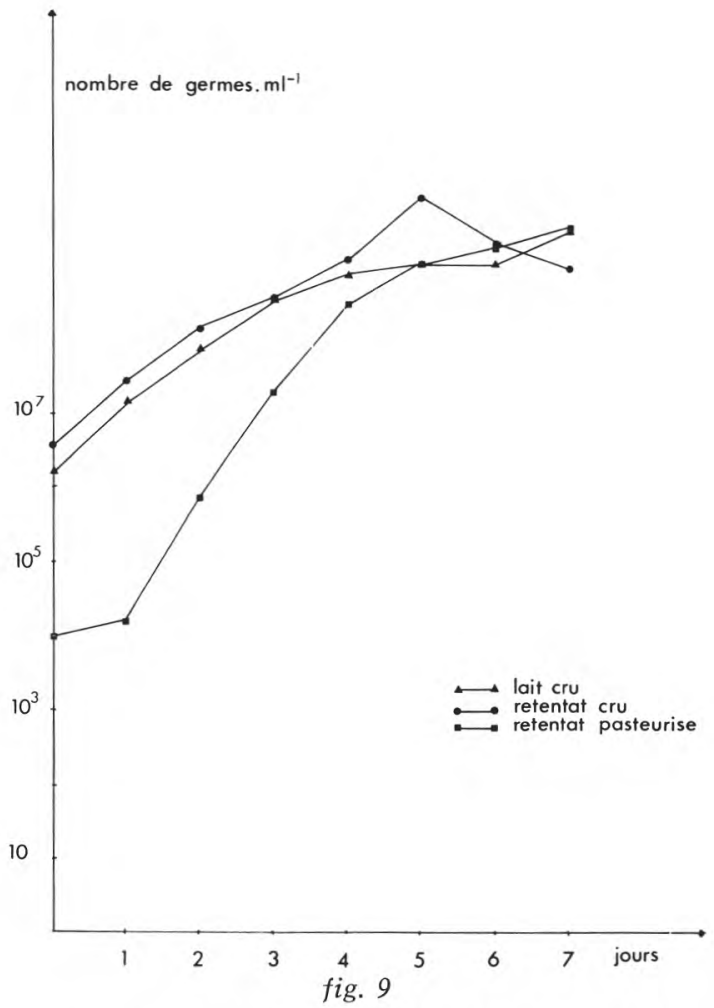

Evolution de la flore psychrotrophe d'un lait et de rétentats 2 cru et thermisé $\left(72^{\circ} \mathrm{C} \pm 1^{\circ} \mathrm{C} / 15 \mathrm{~s}\right)$ au cours de la conservation à $6^{\circ} \mathrm{C}$.

dans le mélange des traites était lente : 0,5 log en $4 \mathrm{j}$, soit une population moyenne de l'ordre de 5000 germes/ml dans les deux séries d'expérimentations.

La flore psychrotrophe des laits crus produits était en moyenne dans les deux séries d'expérimentations de 12000 germes $/ \mathrm{ml}$. Son évollution au cours de l'opération d'ultrafiltration était tout à fait similaire à celle de la flore aérobie mésophile (multiplication par un facteur $2,8)$ mais sa destruction par le traitement thermique du rétentat était supérieure (réduction décimale égale ou supérieure à 3). La croissance observée était de l'ordre de $0,5 \mathrm{log} / \mathrm{j}$ pour les deux séries d'expérimentations. Les populations moyennes atteignaient après $96 \mathrm{~h}$ de conservation dans le mélange de quatre traites (fig. 10) ou de huit traites (fig. 11) respectivement 3000 et 4000 germes $/ \mathrm{ml}$, soit une valeur inférieure à celle de la flore aérobie mésophile. 


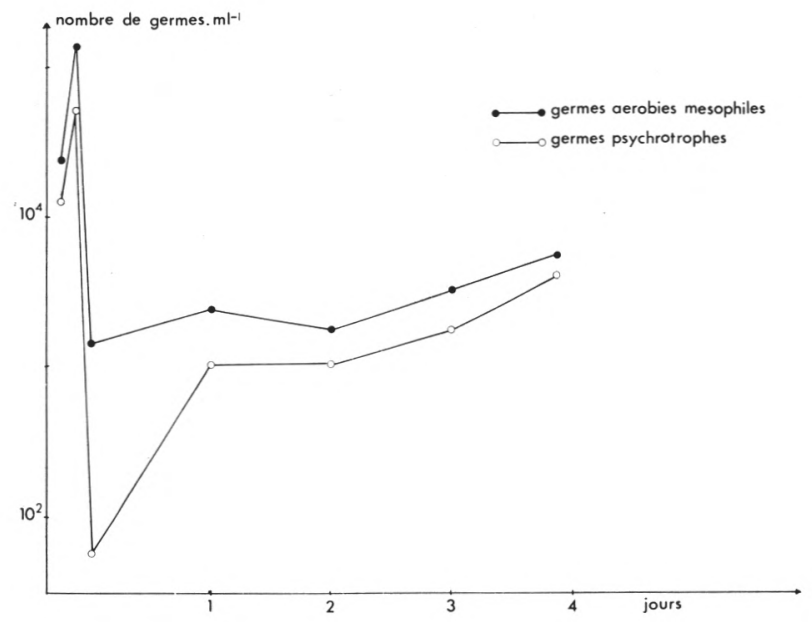

fig. 10

Moyenne logarithmique

Evolution bactériologique d'un mélange de rétentats 2,0 thermisés $\left(72^{\circ} \mathrm{C} \pm 1^{\circ} \mathrm{C} / 15 \mathrm{~s}\right)$.

Accumulation de 4 traites ( 1 traite/j).



Moyenne logarithmique

Evolution bactériologique d'un mélange de rétentats 2,0 thermisés $\left(72^{\circ} \mathrm{C} \pm 1^{\circ} \mathrm{C} / 15 \mathrm{~s}\right)$.

Accumulation de 8 traites $(2$ traites $/ \mathrm{j})$. 


\section{DISCUSSION}

\section{Développement bactérien au cours de l'ultrafiltration}

Les flores aérobies mésophiles et psychrotrophes des rétentats crus représentent 1,6 à 4 fois celles des laits sortant de la machine à traire. La moitié de cette multiplication n'est qu'apparente, elle est due à l'opération d'ultrafiltration elle-même : réduction de 50 p. 100 du volume du lait avec rétention des micro-organismes par les membranes. La multiplication réellement dénombrée, en accord avec les résultats obtenus par Kiviniemi et Seuranen [20] et Veillet-Poncet et al. [37.] est vraisemblablement la résultante de plusieurs facteurs agissant de façon opposée.

- Les vitesses de balayage des membranes utilisées en ultrafiltration entraînent un traitement mécanique non négligeable du lait cru au niveau des pompes. Il peut en résulter une désagrégation des amas microbiens rompus jusqu'au stade diplocoque $[10,8]$, ce qui en conséquence accroît d'environ 50 p. 100 la quantité de germes susceptibles de former des colonies.

- La concentration par ultrafiltration des agents antibactériens (lactoperoxydase - agglutinines, etc.) doit au contraire limiter le développement d'une partie de la flore, surtout si cette opération est réalisée dans les heures qui suivent la traite [3] ce qui était le cas dans nos expérimentations. Cette stabilisation de tout ou partie des flores aérobies mésophile et psychrotrophe est probablement responsable de l'absence de relation trouvée entre les températures d'ultrafiltration et la croissance de ces flores.

- Enfin, la croissance bactérienne "vraie " résultant de la division cellulaire qui elle, est fonction de la durée de l'opération d'ultrafiltration. En raison de l'appareillage utilisé, cette durée variait entre 60 et $90 \mathrm{~min}$. Il est vraisemblable que, dans les appareillages actuellement en cours de développement, ce temps de séjour du lait cru dans les modules d'ultrafiltration sera réduit à environ 20 à $30 \mathrm{~min}$, en raison du nécessaire fonctionnement en continu de ces appareillages. La qualité bactériologique des rétentats et par voie de conséquence celle des produits ultérieurs devrait se trouver améliorée du fait de cette réduction des temps de séjour.

Sur un autre plan, il est possible d'envisager un couplage des équipements de thermisation et d'ultrafiltration tel que cette dernière opération soit réalisée à une température voisine ou supérieure à $50^{\circ} \mathrm{C}$. Outre le gain de performance qui serait obtenu au niveau des débits de perméation, un tel traitement entraînerait par lui-même une réduction notable des flores aérobies mésophile, psychrotrophe et coliforme. Nous l'étudierons dans un proche avenir. 


\section{Effet du traitement thermique}

La concentration différentielle en protéines et en matière grasse dans la zone de traitement étudié n'a aucun effet protecteur sur la sensibilité au traitement thermique des micro-organismes contaminant le lait cru, comme elle n'a aucune influence sur le développement bactérien [25].

La flore mésophile du rétentat cru est réduite par thermisation dans la proportion de 98 à 99 p. 100 , quel que soit le nombre de germes présents dans le rétentat cru. Ce résultat est tout à fait en accord avec les données de la littérature relatives au lait cru [19]. La réduction observée des flores psychrotrophe et coliforme est, elle aussi, comparable aux réductions observées lors de l'application de traitement thermique semblable au lait cru. Bien que plus élevée que celle observée pour la flore aérobie mésophile, la destruction de la flore psychrotrophe n'est pas totale; quelques dizaines de germes survivent au traitement. Il peut s'agir soit d'espèces thermorésistantes comme cela a été en évidence pour les genres Bacillus, Arthrobacter, Microbacterium et Streptococcus (faecalis) [39], soit de recontaminations post-thermisation comme l'ont suggéré Mourgues et Auclair [28]. Le caractère aléatoire de ces recontaminations dans les circuits de récupération et de refroidissement des échangeurs à plaque semblables à celui existant dans l'appareillage "Microtherm ", serait en assez bon accord avec la variation observée des réductions décimales pour un même traitement thermique $(2,5$ à 3,5$)$. Il découle de ces observations que le nettoyage et la désinfection des équipements réalisant une thermisation soit du lait cru, soit du rétentat cru, au niveau de la production devront être réalisés aussi parfaitement que possible pour minimiser autant que faire se peut les recontaminations en germes psychrotrophes, coliformes, etc., des produits thermisés, ce qui exige d'avoir la possibilité, soit de vidanger complètement le circuit "échange- récupération-refroidissement ", soit de le maintenir sous solution désinfectante.

\section{Conservation au froid}

L'évolution des flores aérobies (mésophile, psychrotrophe et coliforme) observée dans le rétentat cru n'est pas significativement différente de celle observée pour ces mêmes flores dans le lait cru lors de la conservation à basse température, que ce soit dans les conditions contrôlées du laboratoire ou dans les conditions de la pratique au niveau de la ferme. Au strict plan de la qualité de conservation des produits, l'ultrafiltration seule ne présente donc d'autre avantage que la redistribution sur place de l'ultrafiltrat aux animaux et elle ne permet pas de réduire la fréquence des ramassages.

Par contre, la combinaison, sur un lait contenant en sortie de machine à traire de 10 à 20000 germes $/ \mathrm{ml}$, d'une opération d'ultrafiltration comportant une réduction en volume de l'ordre de 50 p. 100 
de ce lait avec un traitement thermique immédiat du rétentat permet d'envisager, avec une large marge de sécurité au plan de la qualité bactériologique du produit ramassé, une collecte bi-hebdomadaire. En effet, une telle combinaison conduit à des teneurs en germes aérobies mésophiles et psychrotrophes, dans un mélange de huit traites, largement inférieures à 10000 germes $/ \mathrm{ml}$. Elle permet en outre d'utiliser de façon optimale le parc de tanks de réfrigération actuellement en place sur les lieux de production, qui est prévu pour un ramassage tous les $2 \mathrm{j}$. Comme pour la seule thermisation du lait, la réduction de la flore psychrotrophe résultant du traitement UF + thermisation dans les produits collectés par les transformateurs, devrait avoir des conséquences favorables au niveau des produits élaborés par les entreprises laitières.

Bien que les croissances bactériennes constatées dans le mélange de rétentats thermisés conservés au froid dans les conditions de la pratique soient faibles : environ $0,25 \mathrm{log} / \mathrm{j}$, soit moins d'un doublement de population par jour (fig. 7 et 8), une conservation plus longue, de 1 semaine par exemple, comme celle envisagée par Zall [40] serait aventureuse. Les évolutions constatées dans les échantillons conservés au laboratoire aux températures de $2^{\circ} \mathrm{C}$ et $4^{\circ} \mathrm{C}$ permettent de prévoir que la teneur en germes aérobies mésophiles et psychrotrophes pourrait avoisiner ou même dépasser la valeur de 100000 germes $/ \mathrm{ml}$ dans un mélange de quatorze traites. La flore psychrotrophe serait dominante et tout réchauffement accidentel du produit (au cours du transport à la laiterie, par exemple) risquerait de l'amener à la valeur dangereuse de $10^{6}$ germes $/ \mathrm{ml}$. Une collecte hebdomadaire nécessiterait en outre une modification drastique du parc actuel de tanks de réfrigération.

La comparaison des développements microbiens observés au cours des conservations à $2^{\circ} \mathrm{C}-4^{\circ} \mathrm{C}$ et $6^{\circ} \mathrm{C}$ des laits et rétentats crus et des rétentats thermisés montre à l'évidence, comme l'ont observé sur le lait cru Auclair [3], sur le rétentat cru Veillet-Poncet et al. [37] et sur les laits pasteurisés Mourgues et Auclair [28], l'impérieuse nécessité d'un refroidissement et d'un maintien des rétentats pasteurisés à température au plus égale à $4^{\circ} \mathrm{C}$. Tout ou partie des effets bénéfiques au plan des transformations ultérieures et des produits offerts au consommateur découlant de cette haute qualité bactériologique des rétentats prétraités à la ferme, risquerait d'être perdu s'il se produisait un relâchement dans la surveillance de la chaîne de froid allant du producteur au lieu de transformation.

Les résultats obtenus au cours de cette étude avec les équipements mis à notre disposition permettent d'affirmer que la combinaison d'une ultrafiltration et d'une thermisation est, dans son principe, réalisable sur le lieu de production. Elle conduit à l'obtention d'une nouvelle matière première laitière à teneur doublée en matière grasse et en protéines, d'excellente qualité bactériologique, autorisant une conservation à la ferme de $4 \mathrm{j}$. De nombreuses études restent à 
entreprendre sur de très nombreux plans notamment en ce qui concerne :

- les conséquences physico-chimiques et biochimiques de ce traitement et d'une conservation prolongée au froid du rétentat thermisé ;

- la mise en continu, l'automatisation, la simplification des équipements pour les rendre accessibles économiquement et pratiquement au producteur lui-même ou à un groupement de petits producteurs ;

- les aptitudes aux diverses transformations de cette nouvelle matière première et la qualité, prise au sens large du terme, des produits finaux ;

- les répercussions économiques éventuelles s'il y avait généralisation de ce traitement.

\section{Ré s u mé}

Grâce à un équipement prototype mis en place dans une ferme disposant d'un troupeau de 50 vaches laitières, les aspects bactériologiques d'un traitement du lait cru, sortant de la machine à traire, combinant une opération d'ultrafiltration, jusqu'à un facteur de concentration au plus égal à 2 , et une opération de thermisation du type pasteurisation H.T.S.T. ont été étudiés.

La concentration par ultrafiltration du lait cru entraînait une multiplication " apparente » par un facteur 2,8 des flores aérobies mésophile et psychrotrophe. La multiplication réelle était de 1,4. Le traitement thermique étudié $\left(72^{\circ} \mathrm{C} / 15 \mathrm{~s}\right)$ entraînait une réduction décimale variant entre 2 et 3 ce qui conduisait à un nombre de germes aérobies mésophiles voisin de 1000 U.F.C./ml dans le rétentat thermisé.

L'évolution des flores microbiennes lors de la conservation des laits crus et des rétentats crus et thermisés dépendait étroitement de la température de conservation $\left(2^{\circ} \mathrm{C}-4^{\circ} \mathrm{C}-6^{\circ} \mathrm{C}\right)$ et de la durée du stockage ( 1 à $7 \mathrm{j}$ ). La durée des « lag-phases » variait entre $24 \mathrm{~h}$ $\left(6^{\circ} \mathrm{C}\right)$ et $72 \mathrm{~h}\left(2^{\circ} \mathrm{C}\right)$. Les croissances bactériennes observées variaient entre $0,2 \mathrm{log} / \mathrm{j}\left(2^{\circ} \mathrm{C}\right)$ et $1 \mathrm{log} / \mathrm{j}\left(6^{\circ} \mathrm{C}\right)$.

L'accumulation de 4 (une traite/j) puis de 8 (deux traites par jour) traites consécutives de rétentats thermisés en tank de stockage classique conduisait, dans le mélange, à des flores aérobies mésophiles de l'ordre de 5000 germes $/ \mathrm{ml}$.

Ces résultats très encourageants, joints à une démonstration de la faisabilité technique de l'opération proposée, permettent d'envisager avec confiance le développement futur d'un tel prétraitement au niveau de la production, sous la réserve de nombreuses autres expérimentations. 


\section{S u m m a ry}

\section{ULTRAFILTRATION AND THERMIZATION OF MILK AT THE FARM BACTERIOLOGICAL ASPECTS}

Thanks to a prototype equipment placed in a farm of fifty dairy cows, bacteriological aspects of a treatment combinizing ultrafiltration to a maximum concentration of 2: 1 and thermization (H.T.S.T. pasteurization) on raw milk leaving the milking machine, were studied. Ultrafiltration gave an "apparent" multiplication of aerobic mesophilic and psychrotrophic flora by a 2.8 factor. True bacterial development was 1.4. Studied heat treatment $\left(72^{\circ} \mathrm{C} / 15^{\prime \prime}\right)$ leaded to a decimal reduction varying from 2 to 3 . Consequently, the total mesophilic count of thermized retentate was $1000 \mathrm{CFU} / \mathrm{ml}$.

Storage temperatures $\left(2^{\circ} \mathrm{C}-4^{\circ} \mathrm{C}-6^{\circ} \mathrm{C}\right)$ had a strong influence on bacterial flora of raw milk, raw and thermized UF retentates as also storage time (one to seven days) did. "Lag-phases» varied from $24 \mathrm{~h}\left(6^{\circ} \mathrm{C}\right)$ to $72 \mathrm{~h}\left(2^{\circ} \mathrm{C}\right)$. Bacterial growthes varied from $0,2 \mathrm{log} /$ day $\left(2^{\circ} \mathrm{C}\right)$ to $1 \log /$ day $\left(6^{\circ} \mathrm{C}\right)$.

Accumulation of 4 ( 1 per day) then 8 ( 2 per day) consecutive milkings of thermized retentates in a classical bulk tank leaded to an aerobic mesophilic flora in the mixture of around $5000 \mathrm{CFU} / \mathrm{ml}$.

These results were discussed under the view of an extension of the proposed farm treatment of milk.

Reçu pour publication en juin 1981.

\section{Bibliographie}

[1] Adams (D. M.), Barach (J. T.) and SPeck (M. L.) (1976). - Effect of psychrotrophic bacteria on raw milk proteins and stability of milk proteins to ultrahigh temperature treatment. J. Dairy Sci., 59, 823.

[2] Alichanidis (E.) and Andrews (A. T.) (1977). - Some properties of the extracellular protease produced by the psychrotrophic bacterium Pseudomonas fluorescens strain. Biochim. Biophys. Acta, 485, 424-433.

[3] Auclair (J.) (1964). - Les substances antibactériennes du lait cru et leur rôle en technologie laitière. 4th Int. Symp. on Food Micro., SIK, Göteborg (Suède).

[4] Auclair (J.) (1979.) - Journée d'étude 1979 de l'IESIEL. Effets des techniques de réfrigération et de ramassage du lait sur la qualité bactériologique. Rev. Lait. Franç., 378, 37-39.

[5] Aylward (E. B.), O'Leary (J.) and Langlois (B. E.) (1980). - Effect of milk storage on cottage cheese yield. J. Dairy Sci., 63, 1819-1825.

[6] BesteR (B. H.) (1978). - The effect of cold storage on the bacterial count of raw milk. South African I. Dairy Tech.

[7] Bockelman (I. Von.) (1974), - Growth of bacteria in raw milk during prolonged cold storage. In XIX Int. Dairy Cong., Vol. E (1974), 402-403. 
[8] Bockelman (I. Von), Eriksson (G.) et Hermansson (A. M.) (1975). - L'ultrafiltration du lactosérum doux. L'influence du traitement sur la croissance microbienne et la solubilité des protéines. Milchwissenschaft, 30, 396.

[9] Chapman (H. R.), Bines (V. E.), Glover (F. A.) and Skudder (P. J.) (1974). Use of milk concentrated by UF for making hard cheese, soft cheese and yogurt. J. Soc. Dairy Tech., 27, 151.

[10] Chopin (A.) (1974). - Résultats non publiés.

[11] Cultoli (J.), Bon (J. P.) et Maubors (J. L.) (1974). - Etude de la viscosité des " rétentats " et des " préfromages " obtenus après traitement du lait par ultrafiltration sur membrane. Le Lait, 538, 481-489.

[12] De Boer (R.) and Hiddink (J.) (1980). - Membrane processes in the dairy industry. State of the art. Desalin., 35, 169-192.

[13] Druce (R. G.) and Thomas (S. B.) (1972). - Bacteriological studies on bulk milk collection: pipeline milking plants and bulk milk tanks as sources of bacterial contamination of milk. J. Appl. Bact., 35 (2), 253-270.

[14] Fallick (G. F.) (1969). - Industrial ultrafiltration. Process Biochem., sept., 29-34.

[15] Fauconneau (G.) (1980). - Symposium IBM : Soyons prêts pour les années 1980, Hambourg (R.F.A.).

[16] F.I.L. (1979). - Monography on the bacteriological quality of raw milk. A - Doc. 47 .

[17] Glover (F. A.), Skudder (P. J.), Stothart (P. H.) and Evans (E. W.) (1978). Reviews of the progress of Dairy Science: reverse osmosis and ultrafiltration in dairying. J. Dairy Res., 45 (2), 291-318.

[18] Hartley (J. C.), Vedanuthu (E. R.) and Clark (W. S.) (1968). - Bacteriological methods for evaluation of raw milk quality. A review. I. Use of bacterial tests to evaluate production conditions. J. Milk Food Technol., 31,315 .

[19] Henderson (J. L.) (1971). - In " Microorganisms in the fluid milk industry ». AVI Pub. Co, Wesport (Connecticut - U.S.A.), p. 56.

[20] Kiviniemi (L.) et Seuranen (A.) (1974). - Microbial growth during the ultrafiltration of sweet whey and skim milk. Kemia-Kemi., 12, 791-795.

[21] Law (B. A.), Sharpe (H. E.) and Chapman (H. R.) (1976). - The effect of lipolytic Gram negative psychrotrophs in stored milk on the development of rancidity in Cheddar cheese. J. Dairy Res., 43, 459.

[22] Letourneur (1980). - Communication personnelle.

[23] Maubors (J.L.) et Mahaut (M.) (1974). - Application de l'ultrafiltration sur membrane dans l'industrie fromagère. Rev. Lait. Franç., 322, 479-484.

[24] Maubois (J. L.), Mocouot (G.) et Vassal (L.) (1969). - Procédé de traitement du lait et des sous-produits. Brevet Français n $\mathrm{n}^{\circ} 2052121$.

[25] Maubois (J. L.) et Mocouot (G.) (1971), - Préparation de fromage à partir de " préfromage " liquide obtenu par ultrafiltration du lait. Le Lait, 508, 495-533.

[26] Maubois (J. L.) and Kosikowski (E. V.) (1978). - Making Ricotta cheese by ultrafiltration. J. Dairy Sci., 61 (7), 881-884.

[27] Maubors (J. L.) (1980). - Ultrafiltration et thermisation du lait à la ferme. Bulletin SITMA, 73-78.

[28] Mourgues (R.) et Auclatr (J.) (1969). - Influence de la température de conservation sur le développement de la flore microbienne du lait pasteurisé. Rôle des bactéries psychrotrophes. Rev. Lait. Franç., 268, 505-523. 
[29] O'ConnoR (F.) (1976). - (Total colony count in bulk milk). Methods for evaluation of bacteriological quality of farm milk supplies. Food Progress, 3 (2), 1.

[30] Otenhajmer (I.) and Mitic (S.) (1971). - Characteristic types of psychrophilic bacteria in raw milk. Mljekarstvo., 21 (4), 74-79.

[31] PaLmer (J.) (1979). - Bulletin IDF A-47 "Contamination of milk from the milking environment», 15-22.

[32] RAY (B.) and SPECK (M. L.) (1978). - Plating procedure for the enumeration of coliforms from Dairy Products. Appl. and Envir. Microbi., 35, 820-822.

[33] Rousseaux (P.), Maubors (J. L.) and Mahaut (M.) (1978). - Protein standardization of milk by ultrafiltration in high solids cheese production. In XX Int. Dairy Cong., vol. E, 794.

[34] Randhahn (H.) (1976). - The flow properties of skim milk concentrates obtained by ultrafiltration. J. Text. Stud., 7, 205-217.

[35] SchingoEthe (D. J.) (1976). - Whey utilization in animal feeding: a summary and evaluation. J. Dairy Sci., 59 (3), 556-570.

[36] Thivend (1977). - Utilisation de l'ultrafiltrat de lactosérum dans l'alimentation du ruminant. Econ. Agri., févr.

[37] Veillet-Poncet (L.), Tayfour (A.) et Millière (J. B.) (1980). - Etude bactériologique de l'ultrafiltration du lait et du stockage au froid du rétentat. Le Lait, LX, 351-374.

[38] VIDON (1978). - Communication personnelle.

[39] Washam (C. J.), Olson (H. C.) and Vedamuthie (E. R.) (1977). - Heat resistant psychrotrophic bacteria isolated from pasteurized milk. J. Food. Protect., 49, 101-108.

[40] ZaLl (1980). - Can cheese making be improved by heat treating milk on the farm? Dairy ind. Int., 45 (2), 28-29, 31-48.

\section{Remerciements}

Les auteurs tiennent à exprimer leurs très vifs remerciements à $\mathrm{M}$. J. Poly, Président Directeur Général de l'I.N.R.A. pour l'aide déterminante qu'il a apportée à la mise en place de cet axe de recherches et son constant intérêt. Ils sont également beaucoup redevables à MM. Mocquot, Auclair et Hermier de leurs suggestions et remarques dans la réalisation du manuscrit. 